\title{
REDE DE SAÚDE DO TRABALHADOR PARA O ESTADO DE SÃO PAULO
}

\author{
Koshiro ОтANI
}

\begin{abstract}
Resumo: O artigo reúne documentos do Ministério da Saúde e contribuições dos membros da Comissão IntraSetorial de Saúde do Trabalhador, criada para integrar e harmonizar o trabalho dos diversos setores da Secretaria de Estado da Saúde de São Paulo que lidam com a questão saúde e trabalho. Esta comissão elaborou o Plano de Ações de Saúde do Trabalhador para o Estado de São Paulo (triênio 2002-2003-2004) que estabeleceu, como principal prioridade, a concepção de um modelo para a área de saúde do trabalhador sob a perspectiva de rede de referências técnicas em assistência, vigilância e formação/capacitação.

Palavras-chave: saúde e trabalho; saúde e setor público; ações de saúde.

Abstract: This article considers various Ministry of Health documents and contributions by members of the Intra-Sectorial Commission on Workers' Health, created to integrate and harmonize the work of various sectors of the São Paulo State Secretariat of Workers' Health to address the issue of health and labor. This commission developed the Action Plan for Workers' Health for the State of São Paulo (for the years 2002-2003-2004), which set as its top priority the creation of a model for workers' health that envisioned a network of technical references for treatment, prevention, and training.

Key words: health and labor; health and the public sector; health initiatives.
\end{abstract}

A Atenção Primária à Saúde (APS) constitui-se na forma de organização de serviços de saúde que vem obtendo consenso mundial como a solução para os diferentes problemas de saúde de diferentes países.

Na década de 80, a Organização Mundial da Saúde sob o lema de "Saúde para Todos no Ano 2000" insistia na necessidade de uma organização de serviços primários em todos os países, e no Brasil várias propostas tomaram forma desde o Prev-Saúde e mais recentemente a do Programa de Saúde da Família (PSF).

Naqueles idos, o campo da APS permanecia indefinido, com atribuições e limites imprecisos, estando claro apenas que devia preocupar-se com os "principais problemas de saúde da comunidade", prestando serviços de "promoção, prevenção, tratamento e reabilitação, necessários para resolver estes problemas" (Declaração de Alma-Ata, aprovada pela $32^{\mathrm{a}}$ Assembléia Mundial de Saúde e $34^{\mathrm{a}}$ Assembléia das Nações Unidas).

De lá pra cá, a definição do campo da APS no Brasil passou por um período de reflexão importante em que se discutiu a ordem de prioridade dos diferentes problemas de saúde do país, bem como o volume de recursos possí- veis e disponíveis. A APS deve ser consoante às condições socioeconômicas e culturais de cada Estado, de cada região e de cada centro urbano, e deve ter grau de complexidade compatível com o grau de desenvolvimento econômico e efetiva demanda social.

Nesse processo, a saúde do trabalhador no Sistema Único de Saúde (SUS) que, embora amparada por abundante jurisprudência, continua ineficiente, diante da incapacidade operacional dos setores que o compõem em razão de problemas de ordem estrutural e conjuntural na construção da área de saúde do trabalhador no SUS ${ }^{1}$ como: - ausência de uma cultura institucional sanitária em face dos problemas decorrentes da relação saúde e trabalho;

- a face intensamente ideologizada da área, que implica resistências de caráter político-partidário nos diversos níveis de gestão;

- a dificuldade implicada na visualização da inserção institucional da área;

- caráter inovador da área confrontado à própria abordagem do setor saúde, em relação a estruturas cristalizadas como Vigilância Epidemiológica, Vigilância Sanitária e setores assistenciais; 
- a alta complexidade da abordagem, tanto no aspecto normativo quanto operacional;

- a deficiência quantitativa e a baixa qualificação dos quadros generalistas do SUS.

\section{SITUAÇÃO ECONÔMICA-SOCIAL NO ESTADO DE SÃO PAULO}

A evolução da população economicamente ativa (PEA) no Estado de São Paulo encontra-se nas Tabelas 1 e 2, nas quais se constata, nas duas últimas décadas, um cres-

TABELA 1

População Economicamente Ativa, segundo Setores de Atividade Econômica Estado de São Paulo - 1980-1999

\begin{tabular}{lrrr}
\hline Setores de Atividade & \multicolumn{1}{c}{1980} & \multicolumn{1}{c}{1989} & \multicolumn{1}{c}{1999} \\
\hline TOTAL & 10.236 .019 & 14.379 .365 & 15.412 .014 \\
Primário & & & \\
Agrícola & 1.175 .002 & 1.038 .360 & 1.151 .325 \\
Secundário & & & \\
Indústria de Transformação & 3.068 .936 & 4.062 .989 & 2.780 .628 \\
Indústria da Construção & 795.313 & 914.237 & 1.112 .013 \\
Outras Atividades Industriais & 134.193 & 143.370 & 131.438 \\
Terciário & & & \\
Comércio & 1.102 .525 & 1.877 .242 & 2.345 .048 \\
Prestação de Serviços & 1.946 .814 & 2.590 .197 & 3.469 .528 \\
Serviços Auxiliares de Atividade Econômica & - & 648.026 & 960.114 \\
Transporte e Comunicações & 486.319 & 637.747 & 743.730 \\
Social & 727.267 & 1.315 .867 & 1.637 .498 \\
Administração Pública & 360.868 & 555.498 & 641.959 \\
Outras Atividades & 438.782 & 595.832 & 438.733 \\
\hline
\end{tabular}

Fonte: Fundação IBGE, PNAD - 1980, 1989, 1999. Anuário Estatístico - Brasil. cimento predominantemente no setor terciário, o de prestação de serviços. Observa-se, também, na Tabela 3 um crescimento de trabalhadores não-contribuintes. Eles representam quase $40 \%$ da PEA.

A Tabela 4 exibe a ocupação das pessoas de 10 anos ou mais de idade, segundo os ramos de atividades e condição de contribuintes, e a Tabela 5 apresenta o baixo rendimento familiar ou mesmo sem rendimento, cuja assistência à saúde depende exclusivamente do SUS.

\section{O PAPEL DA SECRETARIA DE ESTADO DA SAÚDE}

A Coordenação dos Institutos de Pesquisa (CIP), considerando a necessidade de implementar as políticas e ações de saúde do trabalhador no estado de São Paulo, resolveu publicar a portaria que instituiu a Comissão IntraSetorial de Saúde do Trabalhador (São Paulo, 2001).

A comissão tem a finalidade de propor medidas com vista na articulação às políticas e coordenar as ações de saúde do trabalhador na Secretaria de Estado da Saúde, na área da Saúde do Trabalhador, com integração dos setores internos que atuam ou possam influenciar nessa área em suas competências e atribuições, com o objetivo de maior eficácia dessas políticas e ações.

O Secretário de Estado da Saúde referendou a comissão (São Paulo, 2002), sem prejuízo das atribuições dos setores que a compõe, com as seguintes atribuições de: I - propor estratégias e táticas para implantar a Norma Operacional de Saúde do Trabalhador - Nost-SUS (Brasil, 1998); ${ }^{2}$

II - implantar o plano de ações de saúde do trabalhador para o Estado de São Paulo, triênio 2001/2003;

TABELA 2

População Economicamente Ativa (PEA) e Taxas de Crescimento Relativas e Absolutas, segundo Setores de Atividade Estado de São Paulo - 1980-1999

\begin{tabular}{|c|c|c|c|c|c|c|c|c|c|c|}
\hline \multirow{3}{*}{ Setores } & \multicolumn{6}{|c|}{ População Economicamente Ativa } & \multicolumn{4}{|c|}{ Taxas de Crescimento (\%) } \\
\hline & \multicolumn{2}{|c|}{1980} & \multicolumn{2}{|l|}{1989} & \multicolumn{2}{|l|}{1999} & \multicolumn{2}{|c|}{$1980-1989$} & \multicolumn{2}{|c|}{$1989-1999$} \\
\hline & $\mathrm{N}^{\text {os }}$ Absolutos & $\%$ & $\mathrm{~N}^{\text {os }}$ Absolutos & $\%$ & $\mathrm{~N}^{\text {os }}$ Absolutos & $\%$ & Absoluta & Relativa & Absoluta & Relativa \\
\hline Total & 9.636 .019 & 100,00 & 15.479 .373 & 100,00 & 15.412 .014 & 100,00 & & & & \\
\hline Primário & 1.175 .002 & 12,19 & 1.038 .368 & 6,70 & 1.151 .325 & 7,47 & $-11,63$ & $-43,89$ & 10,88 & 11,49 \\
\hline Secundário & 3.398 .442 & 35,27 & 5.920 .596 & 38,25 & 4.024 .079 & 26,11 & 74,22 & 10,58 & $-30,34$ & $-31,74$ \\
\hline Terciário & 5.062 .575 & 52,54 & 8.520 .409 & 55,05 & 10.236 .610 & 66,42 & 68,30 & 3,08 & 20,14 & 20,65 \\
\hline
\end{tabular}

Fonte: Fundação IBGE, PNAD - 1980, 1989, 1999 
TABELA 3

Pessoas de 10 Anos ou Mais de Idade, Ocupadas na Semana de Referência, por Contribuição para Instituto de Previdência no Trabalho Principal, segundo Grupos de Idades

Estado de São Paulo - 1999

\begin{tabular}{|c|c|c|c|c|c|c|}
\hline \multirow{3}{*}{ Grupos de Idade } & \multicolumn{6}{|c|}{ Contribuição para Instituto de Previdência no Trabalho Principal } \\
\hline & \multicolumn{2}{|c|}{ Total } & \multicolumn{2}{|c|}{ Contribuintes } & \multicolumn{2}{|c|}{ Não-Contribuintes } \\
\hline & №s Absolutos & $\%$ & №s Absolutos & $\%$ & №s Absolutos & $\%$ \\
\hline Total & 15.412 .014 & 100,00 & 9.436 .856 & 61,23 & 5.975 .158 & 38,87 \\
\hline 10 a 14 Anos & 154.173 & 100,00 & 9.267 & 6,01 & 144.906 & 93,98 \\
\hline 15 a 19 Anos & 1.297 .295 & 100,00 & 547.522 & 42,23 & 749.773 & 57,77 \\
\hline 20 a 24 Anos & 2.146 .341 & 100,00 & 1.392 .370 & 64,87 & 753.971 & 35,13 \\
\hline 25 a 29 Anos & 1.980 .321 & 100,00 & 1.344 .337 & 67,88 & 635.984 & 32,12 \\
\hline 30 a 39 Anos & 4.129.249 & 100,00 & 2.780 .617 & 67,34 & 1.348 .632 & 32,66 \\
\hline 40 a 49 Anos & 3.255 .742 & 100,00 & 2.091 .594 & 64,25 & 1.164 .148 & 35,75 \\
\hline 50 a 59 Anos & 1.665 .404 & 100,00 & 972.926 & 58,42 & 692.478 & 41,58 \\
\hline 60 Anos ou mais & 778.437 & 100,00 & 295.698 & 37,98 & 482.739 & 62,02 \\
\hline Idade Ignorada & 5.052 & 100,00 & 2.525 & 49,98 & 2.527 & 50,02 \\
\hline
\end{tabular}

Fonte: Fundação IBGE, PNAD - 1999.

TABELA 4

Pessoas de 10 Anos ou Mais de Idade, Ocupadas na Semana de Referência, por Contribuição para Instituto de Previdência, no Trabalho Principal, segundo Ramos de Atividade Estado de São Paulo - 1999

\begin{tabular}{|c|c|c|c|c|c|c|}
\hline \multirow{3}{*}{ Setores de Atividade } & \multicolumn{6}{|c|}{ Contribuição para Instituto de Previdência no Trabalho Principal } \\
\hline & \multicolumn{2}{|c|}{ Total } & \multicolumn{2}{|c|}{ Contribuintes } & \multicolumn{2}{|c|}{ Não-Contribuintes } \\
\hline & №s Absolutos & $\%$ & №s Absolutos & $\%$ & №s Absolutos & $\%$ \\
\hline TOTAL & 15.412 .014 & 100,00 & 9.436 .856 & 61,23 & 5.975 .158 & 38,77 \\
\hline \multicolumn{7}{|l|}{ Primário } \\
\hline Agrícola & 1.151 .325 & 100,00 & 434.939 & 37,78 & 716.386 & 62,22 \\
\hline \multicolumn{7}{|l|}{ Secundário } \\
\hline Indústria de Transformação & 2.780 .628 & 100,00 & 2.205 .284 & 79,30 & 575.344 & 20,69 \\
\hline Indústria da Construção & 1.112 .013 & 100,00 & 358.019 & 32,19 & 753.994 & 67,81 \\
\hline Outras Atividades Industriais & 131.438 & 100,00 & 116.271 & 88,46 & 15.167 & 11,54 \\
\hline \multicolumn{7}{|l|}{ Terciário } \\
\hline Comércio & 2.345 .048 & 100,00 & 1.357 .029 & 57,86 & 988.019 & 42,14 \\
\hline Prestação de Serviços & 3.469 .528 & 100,00 & 1.527 .095 & 44,02 & 1.942 .433 & 55,98 \\
\hline Serviços Auxiliares de Atividade Econômica & 960.114 & 100,00 & 623.204 & 64,90 & 336.910 & 35,10 \\
\hline Transporte e Comunicações & 743.730 & 100,00 & 514.620 & 69,19 & 229.110 & 30,81 \\
\hline Social & 1.637 .498 & 100,00 & 1.378 .072 & 84,16 & 259.426 & 15,84 \\
\hline Administração Pública & 641.959 & 100,00 & 568.658 & 88,58 & 73.301 & 11,42 \\
\hline Outras Atividades & 438.733 & 100,00 & 353.665 & 80,61 & 85.068 & 19,39 \\
\hline
\end{tabular}

Fonte: Fundação IBGE, PNAD - 1999.

III - colaborar na elaboração e atualização de normas técnicas relativas à área de saúde e trabalho bem como no estudo e formulação de propostas relativas à saúde ambiental;

IV - coordenar programas, cursos e projetos de capacitação, treinamento, aperfeiçoamento e educação na área de saúde e trabalho, tanto para trabalhadores dos diferentes ramos de atividades como para técnicos da área;
$\mathrm{V}$ - propor convênios com instituições para fins de aprimoramento de suas atividades;

VI - atuar de forma articulada e integrada com as demais unidades pertencentes ao SUS ou não;

VII - promover e participar de investigações e pesquisas científicas em seu campo de abrangência e criar mecanismos para a divulgação de sua produção técnico-científica. 
TABELA 5

Famílias Residentes em Domicílios Particulares e Valor do Rendimento Médio Mensal, segundo as Classes de Rendimento Mensal Familiar Estado de São Paulo - 1999

\begin{tabular}{|c|c|c|c|c|c|c|}
\hline \multirow{2}{*}{$\begin{array}{l}\text { Classes de Rendimento } \\
\text { Mensal Familiar (1) }\end{array}$} & \multicolumn{3}{|c|}{ Famílias Residentes } & \multicolumn{3}{|c|}{ Valor do Rendimento Médio Mensal (em R\$) } \\
\hline & Total & Urbana & Rural & Total & Urbana & Rural \\
\hline Total & 10.697 .950 & 10.015 .260 & 682.690 & 1.231 & 1.262 & 788 \\
\hline Até 1 Salário Mínimo & 425.436 & 386.664 & 38.772 & 124 & 123 & 126 \\
\hline Mais de 1 a 2 Salários Mínimos & 826.451 & 716.041 & 110.410 & 221 & 222 & 213 \\
\hline Mais de 2 a 3 Salários Mínimos & 1.306 .564 & 1.179 .292 & 127.272 & 343 & 344 & 339 \\
\hline Mais de 3 a 5 Salários Mínimos & 1.973 .756 & 1.816 .151 & 157.605 & 538 & 538 & 535 \\
\hline Mais de 5 a 10 Salários Mínimos & 2.827 .836 & 2.698 .880 & 128.956 & 961 & 962 & 936 \\
\hline Mais de 10 a 20 Salários Mínimos & 1.677 .898 & 1.610 .475 & 67.423 & 1.891 & 1.892 & 1.875 \\
\hline Mais de 20 Salários Mínimos & 1.021 .678 & 1.001 .451 & 20.227 & 5.051 & 5.027 & 6.266 \\
\hline Sem rendimento (2) & 336.044 & 314.134 & 21.910 & - & - & - \\
\hline Sem declaração (3) & 302.287 & 292.172 & 10.115 & - & - & - \\
\hline
\end{tabular}

Fonte: Fundação IBGE, PNAD - 1999.

(1) Exclusive os rendimentos das pessoas cuja condição na familia era pensionista, empregado doméstico e parente de empregado doméstico.

(2) Inclusive as familias cujos componentes receberam somente em beneficios.

(3) Exclusive as familias sem declaração do valor do rendimento.

Nota: Valor do salário mínimo em maio de 1999: $R \$ 136,00$.

Os setores internos da SES-SP, participantes da coordenação, são: Coordenação dos Institutos de Pesquisa (CIP); Divisão de Vigilância em Saúde do Trabalhador do Centro de Vigilância Sanitária (DVST/CVS); Divisão de Doenças Ocasionadas pelo Meio Ambiente do Centro de Vigilância Epidemiológica (Doma/CVE); Instituto Adolfo Lutz (IAL); Centro de Referência em Saúde do Trabalhador (Cerest); Coordenadoria de Planejamento em Saúde (CPS); Coordenadoria de Saúde do Interior (CSI); Coordenadoria de Saúde Regional da Grande São Paulo (CSRGSP); Coordenadoria de Recursos Humanos (CRH); Instituição convidada: Conselhos dos Secretários Municipais de Saúde (Cosems).

\section{ATRIBUIÇÕES DA COMISSÃO INTRA-SETORIAL ESTADUAL}

No campo das ações técnicas, a Comissão Intra-setorial Estadual atua como referência na formulação, informação, articulação, capacitação de recursos humanos, desenvolvimento de estudos e pesquisas e acompanhamento de projetos e programas em saúde do trabalhador.

Nas ações administrativas, supervisiona, controla e avalia qualidade de resultados e grau em que as políticas alcançam seu propósito.

A articulação política com os municípios será feita em conjunto com as Direções Regionais de Saúde (DIR) e
Cosems. As DIRs deverão organizar-se para consolidarse como referência regional nos campos da informação e capacitação, propiciando a articulação e integração entre os municípios na construção de redes de referência e contra-referência nas ações de assistência e vigilância epidemiológica e sanitária.

A coordenação promoverá a articulação necessária com outras entidades e órgãos públicos federais, estaduais e municipais, instituições universitárias, com representações de trabalhadores, de empresários, e pode criar comissões ou grupos de trabalho, permanentes ou provisórios.

\section{PRESSUPOSTOS BÁSICOS}

A saúde do trabalhador no SUS configura-se como um campo do saber que se preocupa com as relações entre o trabalho e o processo saúde e doença, desde a atenção básica até o nível terciário.

Considerando que a atuação da saúde do trabalhador no SUS perpassa em ações de vigilância, informação e assistência, a coordenação, para elaborar seu plano de ações, adotou os pressupostos básicos constantes na Norma Operacional de Saúde do Trabalhador:

- universalidade e eqüidade de acesso a todos os níveis de atenção à saúde;

- integralidade das ações individuais/curativas e coletivas de vigilância em saúde; 
- direito a informação;

- participação e controle da sociedade;

- regionalização e hierarquização;

- critério epidemiológico no estabelecimento de prioridades.

O Plano de ações de saúde do trabalhador, a que se refere o item II da resolução da SES, estabeleceu as seguintes prioridades para o triênio 2002-2003-2004 (Quadro 1).

\section{REGIONALIZAÇÃO DAS AÇÕES DE SAÚDE DO TRABALHADOR}

A complexidade do processo saúde-doença-trabalho exige para o sucesso de uma medida sanitária uma reorientação de valores socialmente definidos bem como uma infraestrutura sólida que a viabilize. Isso significa uma política de saúde, um conjunto de medidas orientadas ideologicamente e que permeiem as instituições sociais públicas e privadas, o que coloca o bem-estar dos trabalhadores como objetivo central.

Nessa análise, uma rede estadual de saúde do trabalhador deve servir à hierarquização dos diferentes serviços a serem oferecidos à clientela, com garantia de acesso aos recursos mais sofisticados, mas com racionalização de sua utilização de forma que se evite as distorções que se observam na ausência de uma rede quando esse acesso fica à sorte do trabalhador.
Em busca da consolidação da área de Saúde do Trabalhador no SUS/SP, um dos desafios que se apresenta é a criação de um modelo regionalizado de ações que possibilite melhor compreensão das realidades locais.

Considerando-se os dispositivos emanados pelo Ministério da Saúde na Norma Operacional de Saúde do Trabalhador (Nost/SUS) e na Norma de Assistência à Saúde (Noas), (Brasil, 2002b), as instâncias da SUS devem-se adaptar às novas exigências para a construção de modelo de saúde do trabalhador para o Estado de São Paulo.

O papel a ser exercido pela comissão intra-setorial em nível central e as Direções Regionais (DIR), em nível regional, é o de estabelecer as políticas e as diretrizes em saúde do trabalhador, disseminando-as aos diversos níveis de complexidade do SUS.

As atribuições dessa instância central e regionais seriam as de normalizações técnicas, as relações intra e intersetoriais, o macroplanejamento, a capacitação de recursos humanos e a consolidação de macroindicadores, bem como o estabelecimento de mecanismos de avaliação, de auditoria e de gestão político-estratégica, segundo o preconizado pela Nost.

Já os municípios ou os consórcios municipais teriam papéis e estrutura hierarquizada definida pela Nost/98, conforme o esquema, a seguir, preparado pela comissão intra-setorial de saúde do trabalhador da SES-SP, com ações de atenção básica, secundária e terciária preconizados pelo Ministério da Saúde.

QUADRO 1

Plano de Ações de Saúde do Trabalhador

Estado de São Paulo - 2002-2004

\begin{tabular}{ll}
\hline Prioridades & \multicolumn{1}{c}{ Responsáveis } \\
\hline Conceber um modelo para a área de saúde do trabalhador sob a perspectiva de uma rede & Comissão \\
Pactuar a implantação de um sistema de informação em saúde do trabalhador & CVE/CVS/Cerest \\
Implantar a Nost nos municípios do Estado de São Paulo & Cosems/CIP/CSI/CSRGSP/CCSS \\
Constituir a comissão interinstitucional em saúde do trabalhador no âmbito dos Conselhos Municipais de Saúde & Cosems/CSI/CSRGSP \\
Implantar a Rede de Cuidados Integrais LER/DORT nos municípios no Estado de São Paulo & Cerest/CSI/CSRGSP \\
Reduzir o número de acidentes do trabalho, acidente típico mais acidente de trajeto no mercado formal & Comissão \\
Reduzir o número de casos de doenças ocupacionais no mercado formal & Comissão \\
Reduzir a mortalidade por acidentes do trabalho em motoristas e na construção civil no Estado de São Paulo & Comissão \\
Reduzir a exposição ao fator risco químico, com ênfase ao benzeno, mercúrio e agrotóxico & IAL/CPS/CVS/CVE/Cerest \\
Banir o amianto & Comissão \\
\hline
\end{tabular}

Fonte: SES. 
Gestão Plena da Atenção Básica - Garantir atendimento de acidente do trabalho e ao suspeito/portador de DP/DT: - realizar ações de vigilância nos ambientes e processos de trabalho;

- notificar os agravos à saúde e dos riscos relacionados com o trabalho;

- sistematizar/analisar dados gerados no atendimento aos agravos à saúde relacionados ao trabalho, de modo que se possa orientar intervenções de vigilância/organização dos serviços;

- alimentar sistemas de informação de órgãos/serviços de vigilância e bases de dados de interesse nacional;

- manter unidade especializada de referência em ST.

Gestão Plena do Sistema Municipal - Emitir laudos/relatórios sobre agravos relacionados com o trabalho ou limitações deles resultantes:

- instituir/operacionalizar sistema de referência para atendimento ao AT e ao suspeito/portador de DP/DT, dando suporte técnico para o estabelecimento da relação do nexo com o trabalho, confirmação diagnóstica, tratamento, recuperação e reabilitação da saúde;

- realizar ações de vigilância nos ambientes e processos de trabalho contemplando levantamento/análise de informações, inspeção sanitária, identificação/avaliação das situações de risco, elaboração de relatórios, aplicação de procedimentos administrativos, investigação epidemiológica;

- instituir/manter cadastro atualização das empresas com identificação dos fatores de risco que possam ser gerados para o contingente populacional, direta/indiretamente a eles expostos;

- manter unidade especializada de referência em ST. ${ }^{2}$

\section{Normas de Assistência à Saúde/Norma Operacional de Saúde do Trabalhador}

Gestão Avançada e Plena do Sistema Estadual - Respeitadas as responsabilidades/ prerrogativas dos Municípios habilitados:

- controlar a qualidade das ações de ST desenvolvidas pelos Municípios conforme mecanismos de avaliação definidos em conjunto com as SMSs;

- definir, com Municípios, mecanismos de referência/contra-referência e outras medidas necessárias para o pleno desenvolvimento das ações de assistência e vigilância em ST;

- capacitar recursos humanos para a realização das ações de ST em seu âmbito de atuação;

- estabelecer rotina de sistematização/processamento/ análise de dados sobre ST gerados nos Municípios e em

QUADRO 2

Ações de Saúde do Trabalhador

\begin{tabular}{|c|c|}
\hline Responsabilidades & Atividades \\
\hline \multicolumn{2}{|l|}{ Atenção Básica } \\
\hline Controle dos Acidentes e Doenças Relacionadas ao Trabalho & $\begin{array}{l}\text { - Identificação das situações de risco no território (cadastro de empresas, ambientes de trabalho, } \\
\text { número de trabalhadores, números de crianças expostas ao trabalho infantil e tipos de agravos). } \\
\text { - Detecção de suspeitos dos agravos relacionados ao trabalho (acidente e doenças } \\
\text { relacionadas ao trabalho). } \\
\text { - Registro ou notificação dos casos de acidentes e doenças relacionadas ao trabalho. } \\
\text { - Encaminhamento para Assistência Especializada. } \\
\text { - Ações educativas junto aos trabalhadores e empresas. }\end{array}$ \\
\hline \multicolumn{2}{|l|}{ Atenção Secundária e Terciária } \\
\hline Controle dos Acidentes e Doenças Relacionadas ao Trabalho & $\begin{array}{l}\text { - Confirmação diagnóstica e registro/notificação. } \\
\text { • Acompanhamento dos casos confirmados. } \\
\text { • Vigilância em Ambientes de Trabalho. } \\
\text { - Ações educativas junto aos trabalhadores e empresas. }\end{array}$ \\
\hline
\end{tabular}

Fonte: Comissão de Saúde do Trabalhador do Ministério da Saúde.

Nota: Esquema aprovado em Reunião no Conselho Nacional de Saúde, 2001. 
seu próprio campo de atuação mais rotina de alimentação regular das bases de dados estaduais e municipais;

- elaborar perfil epidemiológico da ST no Estado para subsidiar programação/avaliação das ações de atenção à ST;

- prestar cooperação técnica aos Municípios para o desenvolvimento de ações de ST;

- instituir/manter cadastro atualização das empresas com identificação dos fatores de risco que possam ser gerados para o contingente populacional, direta/indiretamente a eles expostos;

- manter unidade especializada de referência em ST. ${ }^{2}$

Delineada as diretrizes para a regionalização e definidas as ações, a estratégia seguinte é criar uma grande malha com as seguintes características:

\section{CARACTERÍSTICAS DE UMA REDE DE SAÚDE DO TRABALHADOR}

A organização de uma rede primária de assistência à saúde do trabalhador surge como resposta à preocupação de universalização do acesso aos serviços de saúde, determinada jurídica e legalmente pela Constituição e pela Lei Orgânica da Saúde, e obedece a idéia de racionalização da utilização dos recursos tecnológicos, de vigilância em saúde, de diagnóstico e de tratamento para alcançar este fim.

Consoante aos princípios e diretrizes do SUS, o objetivo primordial dessa rede é perpassar as ações de saúde do trabalhador desde a APS até o nível terciário. Suas características redefinem-se para cada região e para cada comunidade, considerando os recursos disponíveis e os problemas que deve resolver.

\section{Articulação Intra e Inter-setorial}

Um modelo de saúde do trabalhador sob a concepção de uma rede prevê sua integração no Sistema Nacional de Saúde, em que a rede primária seja porta de entrada única e de que ela seja capaz de articular inter-setorialmente.

No Estado de São Paulo, existem várias ordens de estruturas de serviços que atuam nas questões saúde e trabalho:

- a fiscalização das normas regulamentadoras, praticada pela Delegacia Regional de trabalho e emprego e Secretaria de Estado de Relações de Trabalho e Emprego;
- os serviços de segurança e medicina do trabalho (SESMT) de empresas e de planos de saúde;

- os serviços médicos hospitalares e privados;

- os serviços da Fundacentro;

- as empresas particulares de assessorias e consultorias; - rede do SUS.

A concepção sistêmica de rede deve perseguir a idéia de articulação intra e inter-setorial nos diversos níveis do SUS de forma que se estabeleça mecanismos de comunicação entre os setores próprios, entidades e instituições que atuam na questão saúde e trabalho, permitindo-lhes melhor definição de papéis, melhor compreensão das realidades locais atuação conjunta na capacitação, na disseminação de conhecimentos e informações.

\section{Estruturas da Secretaria de Estado da Saúde}

O organograma central dos serviços de saúde do trabalhador na Secretaria de Estado da Saúde (SES) reconhece, formalmente, duas ordens de estruturas de serviços para dar conta das vigilâncias sanitária e epidemiológica, além de um setor do IAL que cuida das questões laboratoriais.

Enquanto a atuação da vigilância sanitária privilegia a formação, a capacitação e a informação a epidemiológica tem se empenhado nas questões dos poluentes ambientais, a assistência aos adoecidos pelo trabalho, a articulação inter-institucional e a formação profissional têm sido executadas pelo Cerest, setor ainda não incluído na estrutura formal da SES.

No âmbito das direções regionais de saúde da SES evidencia-se inadequação dos serviços do Estado para atuar na prevenção e assistência dos acidentes e doenças do trabalho. A estrutura de serviços atual da Secretaria na área da saúde e trabalho, composta pelas vigilâncias, não consegue dar conta dessa demanda, tanto pelos parcos recursos humanos, como pela sua vocação histórica. Associar a vigilância com a assistência, apoiada pelo movimento sindical, é o grande mote que se esboça na idealização da rede estadual de saúde do trabalhador, com práticas intervencionistas nas empresas sob nova fundamentação sanitária.

\section{Participação da Classe Trabalhadora}

Diante da atual inadequação na relação serviços/demanda em saúde do trabalhador e considerando a perspectiva de implantação de uma rede estadual de saúde do traba- 
lhador reconhece-se que para além dos planos técnicos a rede deve priorizar a participação das representações sindicais, procurando divulgar o mais amplamente possível a situação atual e os planos elaborados como alternativa viável.

É a própria classe trabalhadora o setor mais legitimamente interessado na superação desses problemas e sem dúvida a que pode levar a luta em sua defesa de forma mais conseqüente. É pela participação e convivência que a instituição absorve valores que contribuirão em sua redefinição.

A participação da classe trabalhadora pode propiciar um exercício reflexivo que crie em seu seio o dimensionamento real de seus problemas e um engajamento efetivo em sua solução, delineando uma vontade coletiva que dê representação política a essa demanda técnica, contribuindo dessa forma para a definição de uma política de saúde direcionada para a valorização do trabalhador.

A participação da classe trabalhadora deve ser entendida como elemento capaz de catalisar uma mudança, por sua expressão política, e também como elemento capaz de contribuir na definição do novo, diante da legitimidade de seus interesses.

Em essência, uma rede em saúde do trabalhador por pretender a socialização dos recursos do SUS é impulsionada a recuperar valores sociais e coletivos para a prática adequada, o que busca mediante participação trabalhadora.

\section{Recursos Humanos e Materiais}

Diante da diversidade de fatores que influenciam a saúde, é hoje iniludível a necessidade do caráter multidisciplinar da equipe de saúde. A viabilização de uma rede primária está diretamente relacionada ao preparo profissional da equipe de saúde e nesse sentido será necessária uma capacitação adequada de seus recursos humanos.

E, a rede ainda, se pretende eficaz, de grande poder resolutivo, pressupõe a presença de profissionais versáteis capazes de responder a variada gama de problemas que constituam o seu alvo.

A definição de alçada e de recursos, a participação dos técnicos das prefeituras municipais e a característica da equipe de saúde constituem os três vértices de uma pedra angular, cujo brilho fica na dependência da luz que a ilumine: a política de saúde.

A definição de recursos deve considerar a populaçãoalvo em seu universo e garantir acesso aos recursos mais sofisticados a toda população trabalhadora, servindo a rede apenas para racionalizar esse acesso e nunca como forma de negação desse direito, criando as diferenças de um tratamento de pobre e outro de rico.

\section{Descentralização das Ações}

A participação dos municípios deve objetivar a permeabilização da instituição aos interesses da clientela a fim de garantir conseqüência à proposta.

Para que essa rede não seja um simples aparelho de reprodução ideológica, é necessário que essa participação encontre meios para expressar sua criatividade e recuperar seus valores.

A participação dos municípios deve ser um elemento ativo em todos os níveis, planejamento, administração, prestação de serviços e não se restringir ao papel de simples executor.

É estratégica a entrada da Saúde do Trabalhador na Saúde da Família/ Atenção Básica, estabelecendo-se protocolos integrais para o conjunto de agravos, tendo-se como perspectiva o trabalho em rede, sem hierarquia e a tolerância às diferenças.

O modelo estrutural de atenção proposto à Saúde do Trabalhador respeita as áreas de abrangências das Direções Regionais de Saúde (DIR), as microrregião ou município para intervenção.

Os critérios de elegibilidade serão os seguintes: o território, considerando as características demográficas, sociais, econômicas e políticas da população local; a existência de situações de risco e sua expressão sob a forma de agravos relacionados com o trabalho; os recursos tecnológicos disponíveis no setor saúde e nos demais pertinentes à situação.

As estratégias de operacionalização para implementação dessa rede são: pactuação intra e extra-setorial, adequação da infra-estrutura da rede de serviços, capacitação dos profissionais da rede de serviços, equipamento, apoio diagnóstico e insumos, comunicação e marketing social, mecanismos de financiamento.

Capacitação - A proposta de capacitação em Saúde do Trabalhador será organizada para ser utilizada como um dos instrumentos para a qualificação dos profissionais de nível superior que atuam com intuito da reestruturação dos serviços de saúde.

O pressuposto para a realização do curso baseia-se no processo de mudança das práticas sanitárias voltadas para a efetivação do Sistema Único de Saúde, coerente com o 
processo de criação e desenvolvimento de Distritos Sanitários, cuja estruturação tem hoje como estratégia o Programa de Saúde da Família, constituindo-se, portanto, em clientela preferencial os profissionais que atuam nesse programa.

Vigilância em Saúde - A visão de uma vigilância em saúde, que envolve as questões ambientais, remete para um debate vital para uma nova conformação estrutural da área. As estruturas da Secretaria em níveis central e regionais, de executoras, passariam a atuar na normalização de procedimentos operacionais, nas relações inter-setoriais, na capacitação de recursos humanos, na disseminação de conhecimentos e de ações para os municípios em seus diversos níveis de complexidade e de forma de gestão, como os recentes consórcios entre os municípios.

Outro aspecto que deve ser abordado é a sistematização, o processamento e a análise dos dados sobre saúde do trabalhador. Já existem sistemas consolidados como o Sivisa, e outros em andamento como projetos, que devem ser implementados para a melhoria das informações (Figura 1).

\section{FIGURA 1}

Proposta de Organização da Saúde do Trabalhador no SUS-SP

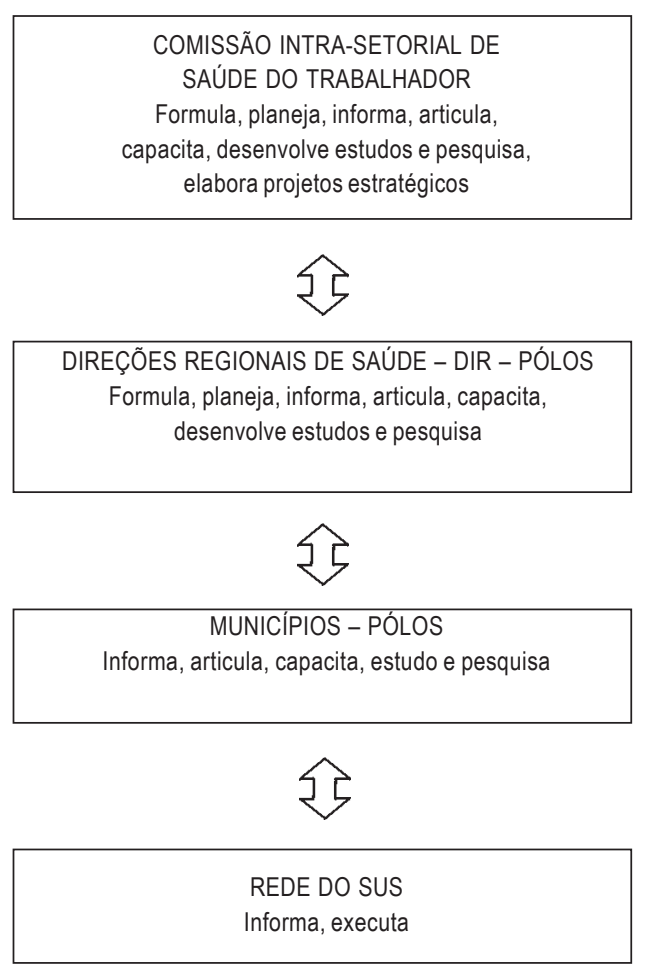

\section{PLANO DE EXPANSÃO DA REDE DE SAÚDE DO TRABALHADOR}

Com base na portaria do Ministério de Saúde que prevê a estruturação de uma Rede Nacional de Atenção Integral a Saúde do Trabalhador, a ser publicada, serão dimensionados os serviços para cada DIR, considerando a necessidade de articular, no campo de ação do SUS, ações de prevenção, promoção e recuperação da saúde dos trabalhadores urbanos e rurais, independentemente do vínculo empregatício e tipo de inserção no mercado de trabalho e considerando o processo de regionalização como estratégia de hierarquização de serviços de saúde e de busca de maior eqüidade, conforme o disposto na Norma Operacional de Assistência à Saúde.

A atenção integral à saúde do trabalhador, com suas especificidades, deve ser objeto de todos os serviços de saúde, consoante com os princípios do SUS, da eqüidade, integralidade e universalidade e considerando a necessidade da criação de mecanismos para o fortalecimento da capacidade de gestão do SUS e a atualização dos critérios de habilitação de estados e municípios.

Hierarquização das Ações Preconizadas pelo Ministério da Saúde e Adaptadas para o Estado de São Paulo:

- Ações na rede de atenção básica e no âmbito do Programa de Saúde da Família (PSF).

- Referências assistenciais nos serviços de média e alta complexidade do SUS.

- Centros de Referência em Saúde do Trabalhador (CRST).

As equipes da atenção básica e do Programa de Saúde da Família serão capacitados para a execução de ações em saúde do trabalhador.

No interior dos serviços existentes de alta e média complexidade, no âmbito de cada Módulo Assistencial, deverão ser organizadas ações especializadas em saúde do trabalhador, cujas atribuições serão estabelecidas em ato específico da comissão intra-setorial.

As direções regionais de saúde (DIR) terão CRST Regional, definidos por ordem crescente de complexidade e distinção de atribuições descritas a seguir.

Os CRSTs Estaduais e Regionais deverão integrar-se entre si e com as referências em saúde do trabalhador desenvolvidas na rede de média e alta complexidade, compatibilizando um Sistema de Informação desenvolvido no âmbito da assistência terciária de média e alta complexidade, a execução de Projetos de Capacitação comuns, 
a elaboração de material institucional e comunicação permanente, de modo que se constitua um sistema em rede nacional.

O controle social da Rede Estadual de Saúde do Trabalhador - mediante participação das organizações de trabalhadores urbanos e rurais -, dar-se-á em todos os níveis - nacional, estadual, regional e municipal, obedecendo aos princípios estabelecidos na legislação vigente.

Serão implantados Centros de Referência Estadual, localizado na capital dos Estados e Centros de Referência Regionais, localizados nas regiões metropolitanas e nas regiões com maior concentração de trabalhadores, com as seguintes atribuições.

\section{CRST Estadual}

- Desenvolver estudos e pesquisas na área de saúde do trabalhador e do meio ambiente, atuando em conjunto com outras unidades e instituições, públicas ou privadas, de ensino e pesquisa ou que atuem em áreas afins à saúde e ao trabalho.

- Promover programas de formação, especialização e qualificação de recursos humanos na área de saúde do trabalhador.

- Dar suporte técnico para o aperfeiçoamento de práticas assistenciais interdisciplinares em saúde do trabalhador, organizada na forma de projetos de intervenção.

- Propor normas relativas a diagnóstico, tratamento e reabilitação de pacientes portadores de agravos à saúde decorrentes do trabalho; promoção de eventos técnicos, elaboração de protocolos clínicos e manuais.

- Atuar em articulação com os Centros de Vigilância Sanitária e Epidemiológica e com unidades e órgãos afins, nas atividades de normalização relativas à prevenção de agravos à saúde decorrentes do trabalho e de vigilância sanitária e epidemiológica em saúde do trabalhador.

- Promover, em conjunto com os órgãos competentes dos municípios, a definição de critérios de: avaliação para controle da qualidade das ações de saúde do trabalhador desenvolvidas no âmbito municipal; referência e contrareferência e outras medidas que assegurem o pleno desenvolvimento das ações de assistência e vigilância em saúde do trabalhador e do meio ambiente; cooperação técnica para o desenvolvimento das ações e pesquisas em saúde do trabalhador e do meio ambiente.

- Produzir informações para subsidiar proposições de políticas na área de saúde do trabalhador.
- Desenvolver programas de educação em saúde sobre questões da relação saúde-trabalho para a população em geral.

- Promover o intercâmbio técnico-científico com instituições nacionais, internacionais e estrangeiras.

- Em conjunto com os gestores estaduais, coordenar o processo de preparação, organização e operacionalização do Programa Estadual de Qualificação Pessoal em Saúde do Trabalhador, estabelecido nessa portaria.

- Em conjunto com os gestores estaduais, coordenar o Programa de Acompanhamento e Avaliação da implantação da Renast.

- Em conjunto com os gestores estaduais, participar do processo de elaboração, implantação e operacionalização do Plano Estadual de Atenção Integral à Saúde do Trabalhador nos municípios, nas diversas regiões do Estado.

- Prestar suporte técnico para os municípios executarem a pactuação regional, a fim de garantir, em toda a área do estado, o atendimento aos casos de doenças relacionadas ao trabalho.

- Participar, no âmbito de cada estado, do treinamento e capacitação de profissionais relacionados com o desenvolvimento de ações no campo da saúde do trabalhador, em todos os níveis de atenção: Vigilância em Saúde, PSF, Unidades Básicas, Ambulatórios, Pronto-Socorros, Hospitais Gerais e Especializados.

\section{CRSTs Regionais}

- Suporte técnico especializado para a rede de serviços do SUS efetuar o atendimento, de forma integral e hierarquizada, aos casos suspeitos de Doenças Relacionadas ao Trabalho, para estabelecer a relação causal entre o quadro clínico e o trabalho.

- Suporte técnico especializado para a rede de serviços do SUS efetuar o diagnóstico e o tratamento das Doenças Relacionadas ao Trabalho, o que inclui a realização de exames complementares, e que pode incluir vistorias sanitárias aos locais de trabalho.

- Suporte técnico especializado para a rede de serviços do SUS efetuar o registro, notificação e relatórios sobre os casos atendidos e o encaminhamento dessas informações aos órgãos competentes visando ações de vigilância e proteção à saúde.

- Suporte técnico às ações de vigilância, de média e alta complexidade, a ambientes de trabalho, de forma integra- 
da às equipes e serviços de vigilância municipal e/ou estadual.

- Retaguarda técnica aos serviços de vigilância epidemiológica para o processamento e análise de indicadores de agravos à saúde relacionados com o trabalho, em sua área de abrangência.

- Ações de promoção à Saúde do Trabalhador, incluindo ações integradas com outros setores e instituições, como Ministério do Trabalho, Previdência Social, Ministério Público, entre outros.

- Participar, no âmbito de seu território de abrangência, do treinamento e capacitação de profissionais relacionados com o desenvolvimento de ações no campo da saúde do trabalhador, em todos os níveis de atenção: PSF, Unidades Básicas, Ambulatórios, Pronto-Socorros, Hospitais Gerais e Especializados.

Os recursos humanos dispostos em cada equipe dos CRSTs deverão ser dimensionados e pactuados na bipartite, com parâmetros mínimos de composição e capacitados para o exercício das ações de saúde do trabalhador.

A proposta de um modelo sob a concepção de rede em saúde do trabalhador, se de um lado tem sido recebido com entusiasmo pelos que militam na área, invariavelmente, de outro, há as reservas do ceticismo em relação às instituições oficiais das quais as pessoas sempre têm um rosário de experiências negativas. Ao lado do ceticismo há também a visão paternalista da instituição a dificultar a organização da participação da classe trabalhadora, o que muitas vezes deixa um ranço servil a essa participação. Enquanto o ceticismo deriva da postura centralizadora e autoritária das instituições oficiais nos últimos anos, o servilismo é reminiscência do período populista de aparelhamento político das instituições e que hoje anda sendo alimentado por setores que se intitulam reformadores.

Ambos os desvios só poderão ser superados pela democratização da instituição. Tem-se claro que esse modelo, ora proposto, embora fundamentado teoricamente, apóia-se muito mais em vontade de transformar. Por isso mesmo, espera-se que ele se constitua em mais uma contribuição a acelerar o início de uma nova era para a saúde do trabalhador no SUS-SP a que por ora apenas esboça seus contornos.

Finalmente, julga-se pertinente a inclusão neste artigo da Carta de São Paulo pela efetiva implantação das ações de saúde do trabalhador no SUS deliberada no II Encontro Estadual para Implantação da Nost/Noas, realizado em 22 de abril de 2002, em São Paulo:
"No bojo da Reforma Sanitária Brasileira, a Saúde do Trabalhador surgiu como uma nova forma de apreender e intervir na relação trabalho-saúde e introduziu na Saúde Pública, a atenção a uma parcela da população estimada em mais de 108 milhões de pessoas inseridas na multiplicidade e diversidade dos ambientes e processos de trabalho desenvolvidos no país.

Instrumentos legais e operacionais vêm conformando, desde a promulgação da Constituição Federal de 1988, o arcabouço que fundamenta o campo da Saúde do Trabalhador, ressaltando-se a Lei oㅜ 8.080/90 (Lei Orgânica da Saúde), as Normas Operacionais Básicas 01/93 e 01/96, a Norma Operacional de Saúde do Trabalhador (Nost) e a Instrução Normativa de Vigilância em Saúde do Trabalhador no SUS, editadas pelo Ministério da Saúde, e em São Paulo, a Constituição Estadual, as Leis no 9.505/97 (que disciplina as ações e serviços de Saúde do Trabalhador no SUS) e no 10.083/98 (que aprova o Código Sanitário do Estado).

Em 24 de março de 2000, realizou-se o I Encontro Estadual para Aplicação da Nost-SUS. Desde então, algumas ações vêm sendo desenvolvidas pelos responsáveis pela implantação das ações de Saúde do Trabalhador no âmbito das esferas estadual e municipal do SUS, porém não logrando efetivar a operacionalização das ações e serviços que a relevância da área requer.

As Normas Operacionais da Assistência à Saúde (NOAS-SUS 01/01 e 01/02), editadas pelo Ministério da Saúde, ampliam as responsabilidades dos municípios na Atenção Básica e atualizam os critérios de habilitação dos Estados e municípios. No entanto, na medida em que apresentam diretrizes para o prosseguimento do processo de descentralização, baseadas na estratégia da regionalização, não contemplam as ações de Saúde do Trabalhador entre as responsabilidades assumidas pelos gestores estaduais e municipais, como também não as incluem nos elencos de procedimentos a serem acrescentados à Atenção Básica e ao componente mínimo da média complexidade ambulatorial.

Os gestores e gerentes das esferas estadual e municipal do SUS, os profissionais do campo da Saúde do Trabalhador e os representantes dos demais segmentos da sociedade presentes ao II Encontro Estadual para a Implantação da NOST/NOAS, realizado no dia 22 de abril de 2002 no Centro de Convenções Rebouças, em São Paulo, preocupados com a insatisfatória cobertura e organização das ações e serviços de Saúde do Trabalhador no Estado e com o objetivo de criar condições para que a 
implantação do preconizado na NOST-SUS ocorra de maneira efetiva, aprovam as seguintes propostas a serem encaminhadas aos órgãos de deliberação e execução do SUS:

- Inclusão das ações contidas na NOST-SUS entre as responsabilidades dos Estados e municípios que assumirem as formas de gestão indicadas na NOAS-SUS 01/01 e 01/02.

- Inclusão da comprovação de capacidade para o desenvolvimento das ações contidas na NOST-SUS como requisito para a habilitação dos Estados e municípios nas formas de gestão indicadas na NOAS-SUS 01/01.

- Aprovação pela Comissão Intergestores Tripartite dos critérios que comporão o Índice de Valorização de Resultados em Saúde do Trabalhador, referido na NOST-SUS, a ser repassado fundo a fundo, aos Estados e municípios. Neste caso, para os municípios, deverá ser utilizado como parâmetro a "Matriz do Modelo de Organização da Atenção à Saúde do Trabalhador para o SUS Municipal", contida na Recomendação no 5 , de 14 de setembro de 2001, do Conselho Nacional de Saúde.

- Liberação de recursos do FAEC (Fundo de Ações Estratégicas do Ministério da Saúde) para a implantação e implementação das redes estaduais de Saúde do Trabalhador.

- Implantação de um componente em Saúde do Trabalhador nos sistemas de informação em saúde de âmbito nacional, que permitam conhecer a realidade de saúde da população trabalhadora, intervir nos fatores determinantes de agravos à saúde nos locais de trabalho, visando eliminálos ou, na sua impossibilidade, atenuá-los e controlá-los; avaliar o impacto das medidas adotadas para a eliminação, atenuação e controle dos fatores determinantes de agravos à saúde e subsidiar a tomada de decisões dos órgãos competentes, nas três esferas de governo.

- Instituição da Comissão Intersetorial de Saúde do Trabalhador, com a participação de entidades que tenham interface com a área, subordinada ao Conselho Estadual de Saúde, com a finalidade de assessorá-lo na definição de políticas, no estabelecimento de prioridades e no acompanhamento e avaliação das ações. Os municípios deverão criar comissões equivalentes no seu âmbito".

\section{NOTAS}

Agradecimentos especiais pela colaboração dos membros da comissão na preparação deste artigo: Aparecida Vieira de Melo; Clelia O S Pedrosa; David Braga Jr.; Elba Pinheiro de Almeida Custódio; Elmir S. Cardim Filho; João Aquino Filho; José Carlos do Carmo; José Geraldo Conceição; Lúcia Toledo; Maria Luiza Rebouças Stucchi; Maria Maeno; Marlene Castanho; Nivaldo D. Teixeira; Paula Pozzi; Paulo Tiglea; Rodolpho Repullo Jr.; Telma de Cássia.

1. Texto para debate - Luiz Carlos Fadel de Vasconcellos - assessor da Coordenação de Saúde do Trabalhador (Cosat) do Ministério da Saúde.

2. Esquema preparado pela Comissão intra-setorial de saúde do trabalhador.

\section{REFERÊNCIAS BIBLIOGRÁFICAS}

BRASIL. Portaria do MS n.3.908/98, Diário Oficial [da] República Federativa do Brasil, Brasília, DF, 10 nov. 1998, n.215-E, Seção 1, p.17.

SÃO PAULO (Estado). Portaria CIP n.12. Diário Oficial do Estado, 31 out. 2001.

SÃO PAULO (Estado). Resolução SS-51 de 19 de abril de 2002. Diário Oficial do Estado, 20 abr. 2002.

SÃO PAULO (Estado). NOAS-SUS fev. 2002, aprovada pela Portaria GM-MS n.373, de 27 de fevereiro de 2002. Diário Oficial de Estado, fev. 2002.

Koshiro Otani: Médico do Trabalho, Coordenador da Comissão Intrasetorial de Saúde do Trabalhador da Secretaria de Estado da Saúde (otani@saude.sp.gov.br) 